\title{
STEREOCHEMISTRY IN THE CYCLIZATION OF O-AZIDOPHENYLALKENES TO INDOLES
}

P. A. S. Smith*, C. D. Rowe, and D. W. Hansen, Jr.

Department of Chemistry, University of Michigan, Ann Arbor, Michigan 48109

Abstract: Cis- and trans-o-azidostilbene, $\underline{0}$-azido- $\beta$-methylstilbene, and ( $\underline{Z})$-o-azido$\beta$-deuteriostyrene give indoles on thermolysis by mechanism involving attack by a nitrene directly on the $\beta$ carbon atom, and not by insertion.

Aryl azides have been observed to insert in saturated C-H groups intermolecularly, and more generally intramolecularly to an or tho substituent when thermolyzed. ${ }^{1}$ With an alkenyl ortho substituent, the result is ring closure to form an indole. ${ }^{2}$ It has not been clear, however, whether that reaction involves attack of a singlet nitrene (2) on the proximal $\mathrm{C}-\mathrm{H}$ bond or on the $s p^{2}$ carbon, or whether it may be abstraction of the proximal $\mathrm{C}-\mathrm{H}$ by a triplet nitrene to produce a diradical $(\underline{3})$ that collapses to an indole.

The parallel reaction by deoxygenation of a nitro group to generate the nitrene has also been studied. ${ }^{3}$ Indoles are obtained. The reaction succeeds whether or not the alkenyl group presents a cis-hydrogen to the nitrene, and if both positions on the $B$ carbon are substituted, one of the groups migrates from it to the erstwhile $\alpha$ carbon (which becomes $\mathrm{C}-3$ of the indole). These facts clearly indicate attack directly on the $\beta$ carbon rather than on the $\mathrm{B} \mathrm{H}$ or $\mathrm{C}-\mathrm{H}$ bond. However, there is some uncertainty about whether the reaction necessarily involves a true nitrene, for in one instance, an N-hydroxyindole was obtained. ${ }^{4}$ Its formation implies that ring closure can occur before deoxygenation to a nitrene has taken place.

We have prepared cis- and trans-o-azidostilbenes $\left(\underline{I}, Z_{2}=N_{2}\right)$ from the known bromo compounds, using the reaction of the Grignard reagents with toluenesulfonyl azide. ${ }^{5}$ The trans isomer ( $44 \%$ yield) had $\mathrm{mp} 95.5-96^{\circ} \mathrm{C}$, and the cis isomer $(48 \%)$ was obtained as an oil. The IR spectra of the trans isomer $\left(970 \mathrm{~cm}^{-1}\right)$ and the cis isomer (920 and $\left.775 \mathrm{~cm}^{-1}\right)$, and the NMR spectra [trans $\delta 6.75(\mathrm{~s})$, cis $\delta 6.49(\mathrm{~s})]$ were consistent with the assigned geometries.

Thermolysis of the trans compound in boiling decalin gave 2-phenylindole (4), mp and mixture mp $188-189^{\circ} \mathrm{C}$, in $88 \%$ yield. The same treatment of the cis isomer also gave 2phenylindole, but in only $18 \%$ yield, along with intractable tar (IR $337 \mathrm{~cm}^{-1}$; NMR $1-2.8$ and 6.8-8.0, broad envelopes).

We originaliy interpreted these results to imply stereospecificity on the assumption that the small amount of ring closure may have been due to some isomerization of the azidocis-stilbene during the reaction; this interpretation is not consistent with the results from o-nitrostilbenes, if both reactions involve nitrenes, and implies that the cyclization 
involves attack on the $\beta$ hydrogen or $\mathrm{C}-\mathrm{H}$ bond, which therefore must be cis to the azide (nitreno) function.

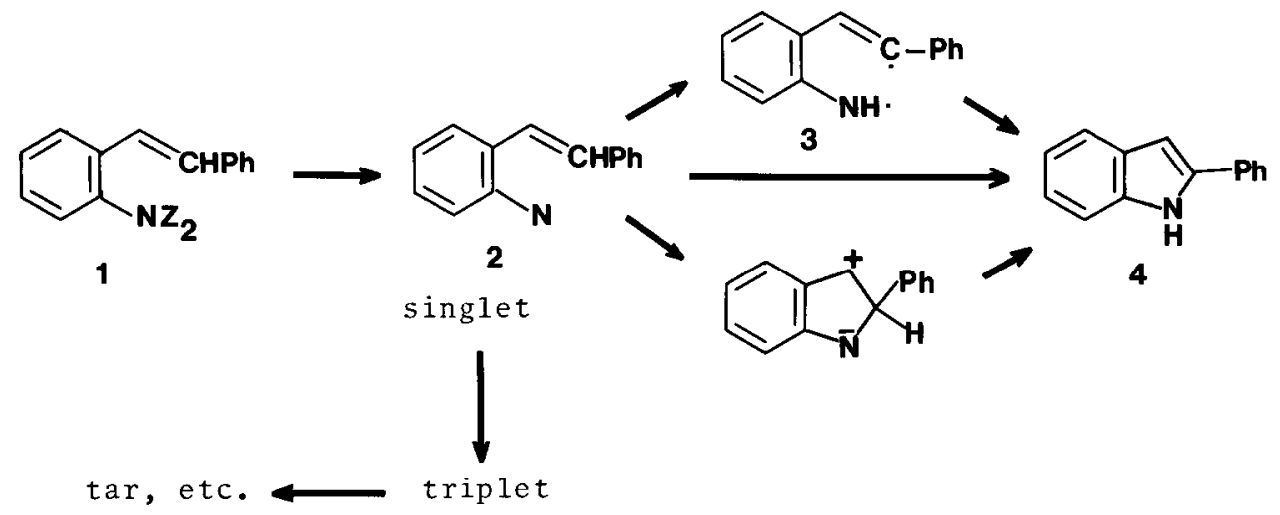

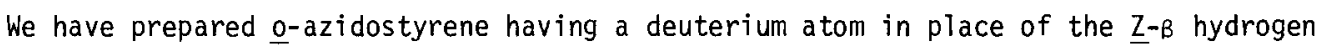
(20\% yield from $\underline{0}$-bromostyrene- $(\underline{Z})-\beta-d$, prepared from $\underline{0}$-bromo- $\beta$-deuteriophenylacetylene $(99 \% \beta-d)$, obtained from 0 -bromophenylacetylene by treatment with BuLi followed by $D C l$, and then hydrogenation over $5 \% \mathrm{Pd} / \mathrm{CaCO}_{3}$, in $50 \%$ overall yield). The (Z)-o-bromo- and ( $\mathbf{z}$ )- $\underline{\mathbf{o}}$ azido- $\beta$-deuteriostyrenes polymerized rapidly, and had to be used at once, but they could be characterized by IR and NMR spectra, which were essentially identical to that of (Z)$\beta$-deuteriostyrene prepared analogously; the $(\underline{E})-\beta-H$ NMR signals appeared as simple doublets, without the splitting by $(Z)-\beta-H$ observed in the nondeuterated analogues, and only traces of absorption at the position of the normal (Z) $-B-H$ resonance (ABMX multiplet) were present. Thermolysis in boiling curiene gave indole in 50\% yield, isolated by preparative TLC. The NMR spectrum indicated $67 \%$ deuterium content at $C-2$ (the N-D was completely exchanged with water during work-up). This distribution is not consistent with attack by the nitrene at $\mathrm{H}(\mathrm{D})$ or the $\mathrm{C}-\mathrm{H}(\mathrm{D})$ bond in the product-determining step, but corresponds to $k_{H} / k_{D}=2$, a reasonable value ${ }^{6}$ only if the product-determining step is tautomerization of $2 \mathrm{H}$-indole formed by attack of the nitrene at the $\beta$ carbon. We therefore conclude that the apparent insertion in these reactions is not a direct one, but involves $\mathrm{N}-\mathrm{C}$ bond formation in the initial step, as is believed to be the case for thermolysis of 0 -azidobiphenyls, which form carbazoles via the nitrenes. 7

The large difference in yields of 2-phenylindole from the geometrically isomeric 0 azidostilbenes may be due to the conformational differences between the isomers. When the phenyl and $\underline{0}$-azidophenyl groups are $c i s$, there is steric interference with rotation of the o-nitrenophenyl group, making it difficult for it to engage the $\beta$ carbon. As a result, there is time for intersystem crossing to the triplet nitrene to take place, and formation of the observed tars results.

It appears, therefore, that the mechanism for cyclization of o-substituted styrene derivatives is the same for both the o-nitro and o-azido systems. To confirm this, we 
prepared (E)-0-bromo- $\beta$-methylstilbene from o-bromobenzyl bromide by the Wittig synthesis with acetophenone in $28 \%$ yield, and converted it by the reaction of its Grignard reagent with toluenesulfonyl azide to o-azido- $\beta$-methylstilbene $(\underline{5})$, an oil [NMR $\delta 2.15(\mathrm{~d}, 3 \mathrm{H})$, $6.37(\mathrm{~m}, 1 \mathrm{H}), 6.5-7.2(\mathrm{~m}, 9 \mathrm{H})]$ in $72 \%$ yield. It formed a crystalline iminophosphorane adduct with triphenylphosphine; mp $192-193^{\circ} \mathrm{C}$. Thermolysis of the azide in decalin at $195^{\circ} \mathrm{C}$ for $2 \mathrm{~h}$ gave a complex mixture from which 2-methyl-3-phenylindole (6) was isolated by column chromatography and distillation in low yield, identified by IR and retention time in VPC. Thermolysis of a sample of the azide in the injection port of a gas chromatograph gave 2-methyl-3-phenylindole in a yield of $59 \%$ of the volatile components. This compares with the yield of $77 \%$ reported by Sundberg and Yamazaki ${ }^{3}$ for deoxygenation of the corresponding nitro compound with triethyl phosphite.<smiles>[X][C@H](C)/C(C=C)=C/c1ccccc1N([Z2])c1ccccc1</smiles>

5

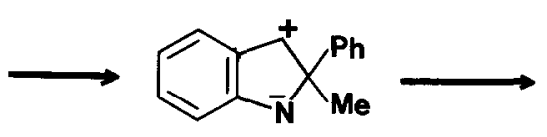

In addition to the indole and the expected o-aminostyrene, two minor products arising from attack on the solvent in the thermolysis of cis-o-azido- $\beta$-deuteriostyrene in cumene are worthy of note. In one ( $12 \%$ yield) (MS $\mathrm{m} / \mathrm{e} 252)$, the methyl groups of the erstwhile cumene isopropyl group appeared in the NMR as a singlet $(\delta 1.45,6 \mathrm{H})$, and the other resonances $(\delta 6.2-6.8, \mathrm{~m}, 2 \mathrm{H} ; 6.8-7.1, \mathrm{~m}, 8 \mathrm{H})$ were cons istent with the structure of $\mathrm{o}^{-}$ (cumylamino)-B-deuteriostyrene (7), a product of nitrene insertion into the tertiary $\mathrm{C}-\mathrm{H}$ bond. ${ }^{8}$ In the second $(10 \%)$, the isopropyl group remained intact (NMR $\delta 1.2, d, 6 \mathrm{H} ; 2.8-$ $3.0, \mathrm{~m}, 1 \mathrm{H})$, but new vinylic protons and only four aryl protons were evident $(\delta 6.2-6.6$, $\mathrm{m}, 6 \mathrm{H} ; 6.8-7.1, \mathrm{~m}, 4 \mathrm{H}$ ), implying the azepine structure (8) or a position isomer (probably in equilibrium with its azanorcaradiene valence tautomer). Compound 8 had no absorption attributable to $\mathrm{N}-\mathrm{H}$, but 7 showed a broad lump centered at $\delta 3.4$.

Such attack of an arylnitrene on another benzene ring is exceptional. Diarylamines, which are formally insertion products of arylnitrenes into an $\mathrm{Ar}-\mathrm{H}$ bond, have been reported in rare instances. ${ }^{9}$ They are presumably derived from rearrangement of an initially formed azanorcaradiene. ${ }^{10,11}$ The alternative isomerization represented by formation of $\underline{8}$ has been reported for the reaction of sulfonyl azides with aromatic hydrocarbons, ${ }^{12}$ but heretofore has only been encountered with arylnitrenes in the case of deoxygenation of perfluoronitrosobenzene in the presence of benzene or anisole, ${ }^{10}$ and deoxygenation of nitrosobenzene in the presence of benzene in trifluoroethanol. 11 Neither phenyl nor perfluorophenyl azides give rise to azepines by thermolysis or photolysis, however. 10,11 The analogous reaction of aryl carbenes to form tropilidenes is well known. 13 

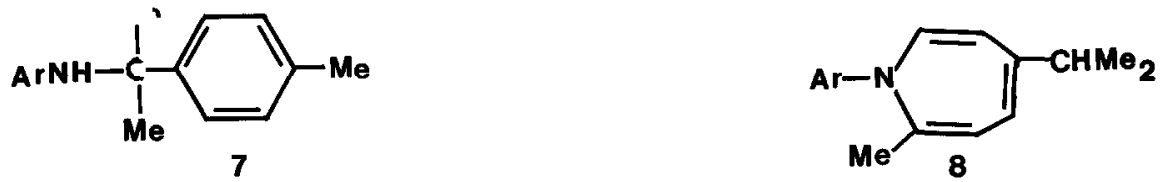

$[$ Ar $=$ o-deuteriovinylpheny 1$]$

The foregoing products provide confirmation of the presumption that nitrenes are involved in thermolysis of $\underline{0}-a l k e n y l p h e n y l$ azides.

Acknowledgment. Financial support for a part of this work by a grant from the $\mathrm{Na}$ tional Science Foundation, GP 15830 , is gratefully acknowledged.

\section{References}

1. P. A. S. Smith, in "Nitrenes," W. Lwowski, ed., John Wiley and Sons, New York, 1970, Chapter 4; R。A. Abramovitch and E. P. Kyba, in "The Chemistry of the Azido Group," S. Patai, ed., John Wiley and Sons, New York, 1971, pp. 256-278.

2. R. J. Sundberg, L.-S. Lin, and D. E. Blackburn, J. Heterocyc1. Chem., 6, 441 (1969).

3. R. J. Sundberg and T. Yamazaki, J. Org. Chem., 32, 290 (1967).

4. R. J. Sundberg, J. Org. Chem., 30, 3604 (1965).

5. P. A. S. Smith, L. B. Bruner, and C. D. Rowe, J. Org. Chem., 34, 551 (1969).

6. This is a minimum value; if some non-deuterated azidostyrene were present in the substrate, correction for it would lead to a correspondingly higher value, but the conclusions, which do not depend on the exact value of $k_{H} / k_{D}$, would not be affected.

7. R. J. Sundberg, D. W. Gillespie, and B. A. DeGraff, J. Am. Chem. Soc., 97, 6193 (1975).

8. Preferential insertion into the tertiary $\mathrm{C}-\mathrm{H}$ of saturated hydrocarbons has been observed by J. H. Hall, J. W. Hill, and H.-C. Tsai, Tetrahedron Lett., 2211 (1965).

9. For example, see J. Ashby, E. F. V. Scriven, and H. Suschitzky, J. Chem. Soc., Chem. Commun., 366 (1972); R. A. Abramovitch, S. R. Challand, and E. F. V. Scriven, J. Org. Chem., 37, 2705 (1972).

10. R. A. Abramovitch, S. R. Challand, and E. F. V. Scriven, J. Am. Chem. Soc., 94, 1374 (1972).

11. R. J. Sundberg and R. H. Smith, Jr., Tetrahedron Lett., 267 (1971).

12. R. A. Abramovitch and V. Uma, J. Chem. Soc., Chem. Commun., 797 (1972).

13. P. A. S. Smith and E. M. Bruckmann, J. Org. Chem., 39, 1047 (1974).

(Received in USA 16 August 1983) 